\title{
Nanostructured Metal Aggregate-Assisted Lasing in Rhodamine 6G Solutions
}

\author{
Valery A. Donchenko ${ }^{1}$, Yuri E. Geints ${ }^{1,2 *}$, Vladimir A. Kharenkov ${ }^{1,3}$, Alexey A. Zemlyanov ${ }^{1}$ \\ ${ }^{1}$ Siberian Physico-Technical Institute, Tomsk, Russia \\ ${ }^{2}$ V.E. Zuev Institute of Atmospheric Optics SB RAS, Tomsk, Russia \\ ${ }^{3}$ Tomsk State University, Tomsk, Russia \\ Email: *ygeints@iao.ru
}

Received August 23, 2013; revised September 25, 2013; accepted October 21, 2013

Copyright (C) 2013 Valery A. Donchenko et al. This is an open access article distributed under the Creative Commons Attribution License, which permits unrestricted use, distribution, and reproduction in any medium, provided the original work is properly cited.

\begin{abstract}
We report on the experimental demonstration of considerable lowering of stimulated luminescence (lasing) threshold in R6G laser dye solution doped with metal agglomerates (Ag, Al, Ni) upon second harmonic of Nd:YAG-laser illumination. Due to strong structural coupling between individual nanoparticles, they may form fractal agglomerates providing an extreme optical pump-field enhancement near their complicated surface. For the first time, we have observed more than twofold increase in lasing efficiency of metal aggregated dye solution as compared to non-agglomerated monomeric metal nanocolloids. No random laser manifestation or surface plasmon-polariton resonances excitations were observed.
\end{abstract}

Keywords: Nano-Composite; Luminescence; Lasing; Metal Agglomerate; Surface Plasmon-Polariton Resonance

\section{Introduction}

One of the most important problems in laser physics is the creation of high-efficiency laser media. A very promising area of researches is related to the development of active media, which is liquid or solid-state composites consisting of a laser-active molecule and nanostructures of various materials $[1,2]$. At present, the studies of laser generation in randomly inhomogeneous media have formed a new area of laser physics called "random lasers" [1]. Laser generation in strongly scattering amplifying medium without any resonator, for the first time, was obtained experimentally by Markushev in 1986 [3], who utilized the powders of crystals containing rare-earth ions. Later, lasing was demonstrated in dye solutions containing strongly scattering $\mathrm{TiO}_{2}$ particles [4,5], and the dyes in the polymer matrix doped with nanoparticles [6].

Laser-gain media based on the "active moleculesnanoparticles" composites are interesting primarily because of the significantly lowering of stimulated emission threshold as compared to that in active medium without nanoparticles. This fact opens a wide opportunity for the creation of highly efficient micro-lasers in optical wavelength range. Up to date, there are two theoretical

\footnotetext{
"Corresponding author.
}

concepts explaining the physical mechanisms of lasing threshold lowering in nanocomposites. The first one deals with active medium doped with metal nanoparticles having marked plasmon-polariton resonances (PPR) in the spectral range of interest [7]. The second concept is developed for active media with nanoparticles that do not exhibit these properties, i.e., plasmon resonances in these nanoparticles are not excited in the spectral range of the pumping radiation and luminescence spectrum of laserdye molecules; however the particles possess strong scattering properties [8].

Accordingly, the physical reasons for the lowering of the threshold of laser generation in these two classes of nanostructured active media are treated differently. In the first type of metal composites, this is due to the large, up to several orders of magnitude, increase in the local optical fields of pumping radiation near the surface of nanoparticles in the conditions of PPR excitation. This increases in its turn the number of excited active molecules. Gold, silver, copper, and some rare-earth compound nanoparticles have strong plasmon resonances in the visible range. At the same time, this is a limiting factor for designing the micro emitters in other spectral ranges where PPRs could not be excited.

The advantage of laser generation in the second class 
of nanoscale composites is gained by the substantial increase of time period, during which the photons of secondary radiation interact with active molecules due to multiple scattering at nanoparticles. The longer is the time that emitted photon travels in an active medium, the higher is the gain of the stimulated emission. This type of lasing is most effectively realized in the regime of diffuse light scattering and is attributed to the "random lasers". The main problem limiting the use of "random lasers" is significantly large energy losses of optical pumping in such strong scattering medium [9].

Numerical calculations carried out by different research groups indicate that near the surface of agglomerated nanostructures with complex surface, there are the areas, where optical near-field intensity can exceed the intensity of incident radiation by several orders of magnitude $[10,11]$ or more, even if no PPRs are excited [12, 13]. So, it is naturally to expect that when a laser dye is doped with agglomerated nanoparticles having plasmon absorption band different than the spectrum of pumping radiation, the yield of dye luminescence and the lasing efficiency may increase.

The purpose of this paper is to test experimentally this hypothesis and to show that just the high-intensity optical fields near the surface of the metal agglomerates may lead to enhanced dye lasing. In order to avoid the manifestation of PPR absorption and laser generation due to multiple random scattering, we used an optically thin layer of the laser dye (rhodamine 6G) solution doped with different metal powders, which can form the complex-type fractal agglomerates and have no PPRs in the spectral range of interest. Our findings show that the intensity of laser stimulated fluorescence in dye-agglomerate colloids is at least twice as high as that with isolated metal nanoparticles.

\section{Experiments}

Our experimental studies were conducting according to the following scheme. The focused optical radiation of Nd:YAG-laser (Lotis TII LS-2132 UTF) with wavelength $532 \mathrm{~nm}$, pulse duration $6 \mathrm{~ns}$, pulse repetition rate 1 $\mathrm{Hz}$, and beam diameter $2 \mathrm{~mm}$ was directed onto the totally reflecting (TIR) prism and then to the horizontal cell with the depth $150 \mu \mathrm{m}$ filled with the colloidal test medium. Optical cell arrangement enabled avoiding possible precipitation of nanoparticles. The signal of pump stimulated dye emission was collected by the collimator and transported by polymer fiber with the diameter of $400 \mu \mathrm{m}$ to the spectrometer (Avaspec-ULS2048L-USB2, spectral range $450-680 \mathrm{~nm}$ ), which was connected to the PC.

We studied the solutions of rhodamine 6G laser dye in ethanol and nanoparticles of $\mathrm{Ag}, \mathrm{Al}$, and Ni prepared by the electro-explosive method in argon atmosphere [14] that produces nanometer different-sized spheres. The estimated diameter of monomeric particles was in the range $10-50 \mathrm{~nm}$. The particles may agglomerate and form the chained clusters with the effective dimensions in the range $100-1000 \mathrm{~nm}$. Single silver nanoparticles with the sizes from 10 to $15 \mathrm{~nm}$ were obtained by chemical procedure [15]. The typical microphotographs of Ag nanoparticles and their aggregates obtained by an electron microscope JESP-II are presented in Figures 1(a)-(d). The absorption spectra of $\mathrm{Ag}, \mathrm{Al}$, and $\mathrm{Ni}$ nanoparticles obtained with spectrometer SOLAR CM 2203 are shown in Figure 1(e).

The phase and structural compositions of metal particles and agglomerates were measured by means of diffractometer (Shimadzu XRD 6000) placed in the BreggBrentano geometry together with the monochromator in the secondary beam. X-ray diffraction patterns of agglomerated nanoparticles of $\mathrm{Ag}$ and $\mathrm{Al}$ are presented in Figures 2(a) and (b). As seen, the measured patterns are in good agreement with the theoretically predicted diffraction pattern. This testifies the purity of used metal particles and absence of metal oxides.

\section{Results and Discussion}

To reveal the role of light multiple scattering on nanoparticles, we measured the optical extinction of tested nanocolloids. Our measurements showed that even for
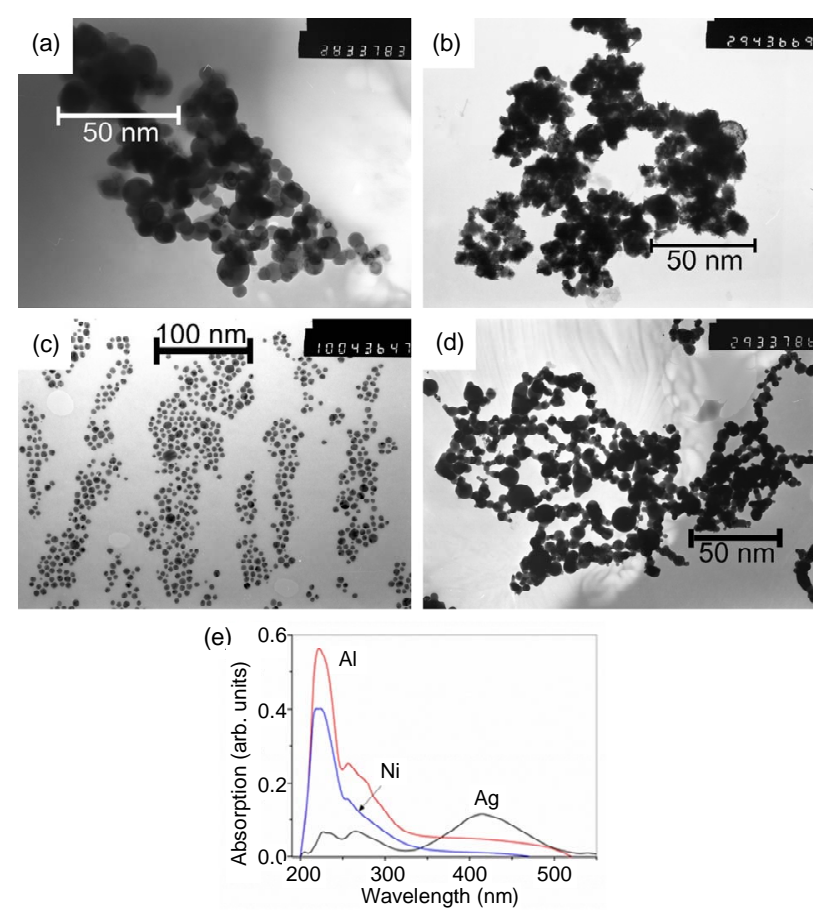

Figure 1. (Color online) Electron microscope images of (a) Ag agglomerates, (b) Al agglomerates, (c) single Ag nanoparticles, (d) Ni agglomerates; (e) absorption spectrums of $\mathrm{Ag}, \mathrm{Al}$, and $\mathrm{Ni}$ agglomerates. 


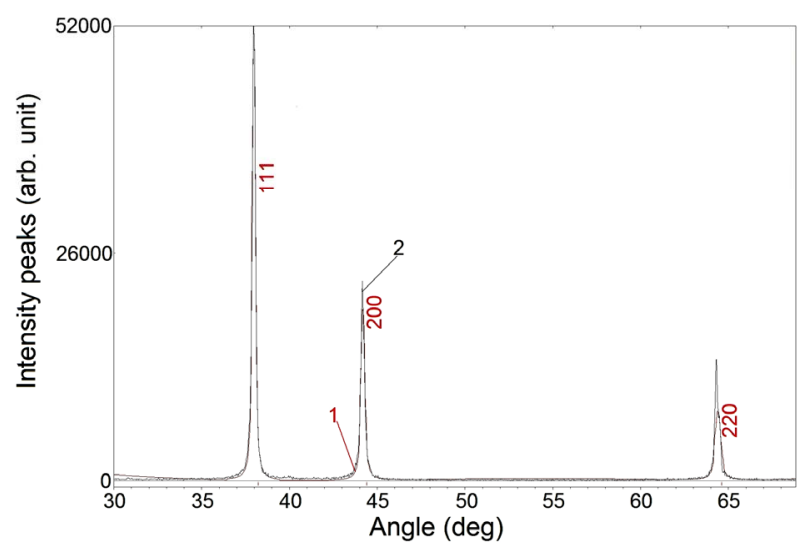

(a)

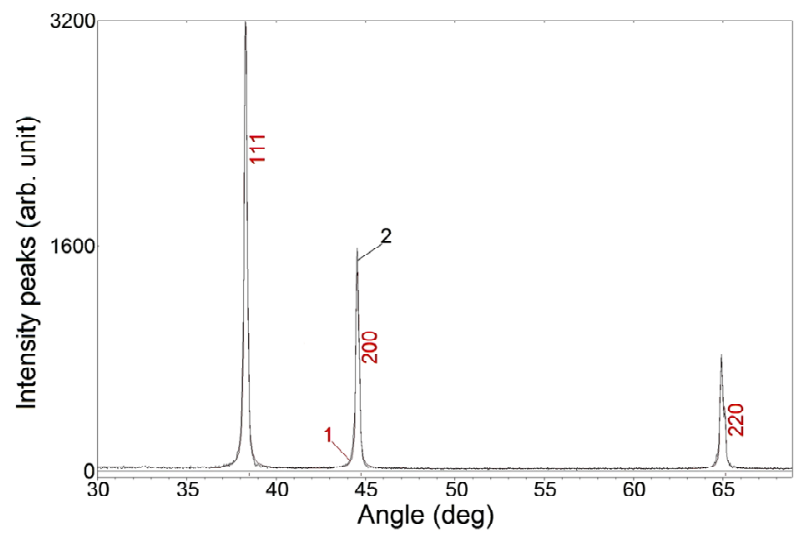

(b)

Figure 2. (Color online) $\mathrm{X}$-ray diffraction patterns of agglomerated (a) Ag and (b) Al nanoparticles: theory (1) and measurements (2).

the cell thickness $630 \mu \mathrm{m}$ and $\mathrm{Ag}$ particle volume concentration up to $9 \%$, the optical transmission of the solutions obeys the Bouguer-Beer law derived in the singlescattering approximation. Calculated optical depth for $140 \mu \mathrm{m}$ thick cell, used in further experiments, filled with $2 \%$ nanostructured metal particle aggregates averages to 0.33 . According to [13], the fraction of multiply scattered radiation in such optically thin heterogeneous medium is negligibly compared to a single scattering. Therefore, the conditions for "random laser" generation in our experiments were not realized.

It is clear that the absorption spectrums of metal nanoparticles that we used in our experiments do not overlap with the pump laser wavelength $\lambda=532 \mathrm{~nm}$, as well as with the absorption and luminescence spectra of pure R6G laser dye or emission spectrum of dye-particles mixture (Figure 3). Consequently, we can suppose that the effect of PPR-supported luminescence in our conditions was not realized. Additionally, we have to notice that the maximum of pure R6G dye emission spectrum and the maximum of dye-nanoparticles mixture spectrum are located at the same wavelength $(\sim 565 \mathrm{~nm})$ irrespective of particles material and structural morphology. This suggests once more that under our experimental conditions the "random laser" mode was not observed.

In Figure 4 we plotted the stimulated emission intensity maxima measured in several dye mixtures with monomeric metal nanoparticles and aggregates of particles by varying the energy $E$ of pumping laser. As can be seen, when pumping is weak, the luminescence signal grows linearly with the increase in E. Starting from the certain pump energy (marked by arrows in the figure), the type of the presented dependences changes to near exponential that suggests the beginning of stimulated luminescence, which also manifests itself in the narrowing of the emission spectrum (see, Figure 3).

From this figure, it is evident that at the fixed pumping energy the intensity of dye lasing is manyfold enhanced by doping the active medium with metal nanoparticles.

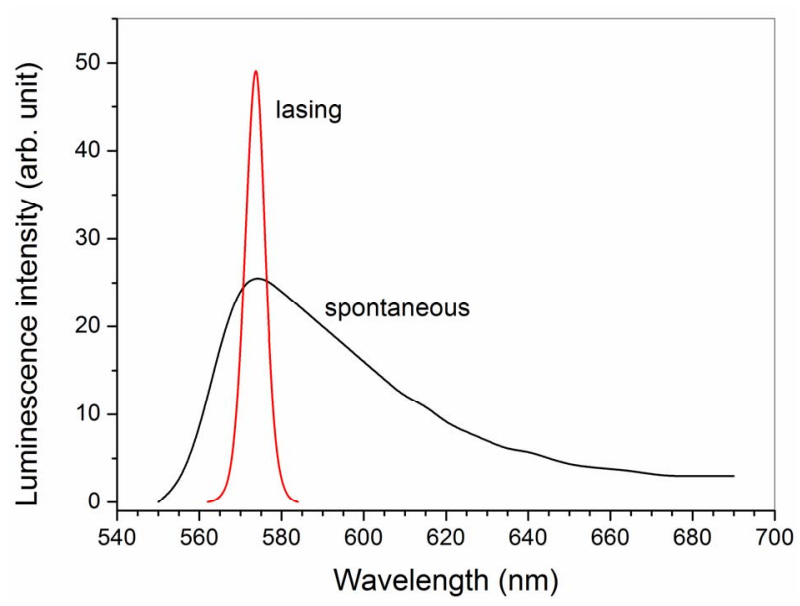

Figure 3. (Color online) Typical spectra of spontaneous luminescence and lasing in R6G-Ag-nanoparticles mixture.

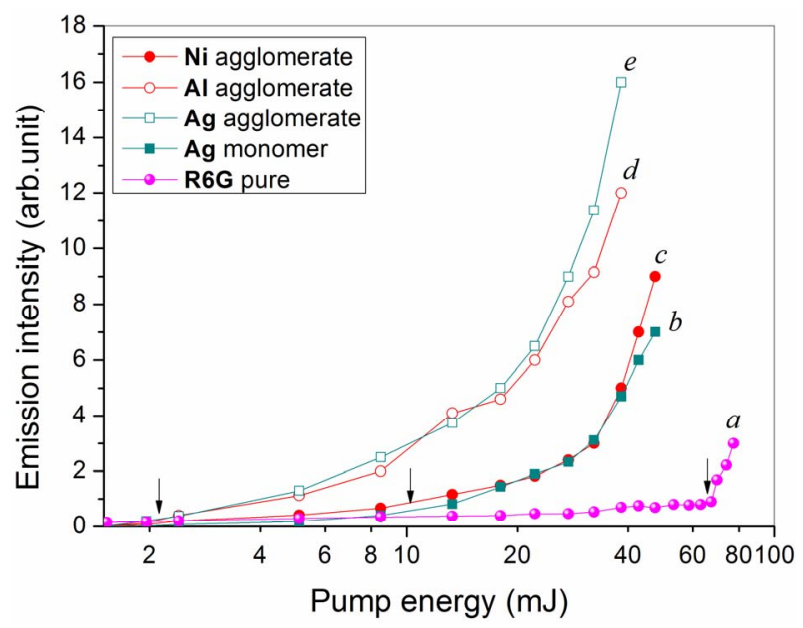

Figure 4. (Color online) Luminescence intensity versus laser pumping energy in (a) pure R6G dye, (b) R6G mixture with single Ag particles, (c-e) $2 \%$ mixture with agglomerated nanoparticles of (c) Ni, (d) Al, (e) Ag. 
The mixtures with single $\mathrm{Ag}$ and $\mathrm{Ni}$ particles reveal similar stimulated emission efficiency up to the pump energy $60 \mathrm{~mJ}$. The maximal increase of the stimulated luminescence intensity is observed in the mixture with agglomerated particles. It is important to note that the generation efficiency of R6G solutions with agglomerates of $\mathrm{Ag}$ and $\mathrm{Al}$ nanoparticles is higher than the $\mathrm{Ni}$ agglomerates and single Ag nanoparticles.

Thus, our results show that the efficiency of lasing in mixtures of R6G with agglomerated nanoparticles is much higher than the efficiency of laser generation in solutions with single Ag nanoparticles. So far as the implementation of strong scattering conditions in our experiments were prevented (i.e., the "random laser" generation) and there were no marked PPR absorption in metal particles, we can suppose that the enhancement of the stimulated luminescence in nanostructured agglomerated laser dye as compared to the generation in solutions with single Ag nanoparticles is provided by higher intensities of the local optical fields acting as the exciting source near the complicated surface of agglomerated nanoparticles. Our findings are in correspondence with the results of the theoretical calculations $[11,12,16]$, which show that the local optical intensity near coupled metal nanoparticles illuminated by a laser field sharply increases with the decreasing of the interparticle gap.

Interestingly, the lasing efficiency in solutions with agglomerates of $\mathrm{Ag}$ and $\mathrm{Al}$ was almost the same, while the agglomerates of Ni nanoparticles gave the worst results among all investigated agglomerates. As was pointed above, due to the strong magnetic properties of Ni nanoparticles, we were not able take pictures of these particle agglomerates with an electron microscope. However, because the Ni nanoparticles were produced by electro explosive procedure similarly to other conglomerates, we can expect that the morphology of Ni nanoparticles does not differ from the morphology of other agglomerates. In this sense, the worst lasing support properties of $\mathrm{Ni}$ aggregates can be attributed to their specific electro physical and optical properties. This issue requires a further study.

In conclusion, we have experimentally compared the stimulated luminescence of the Rhodamine 6G laser dyes (concentration $10-3 \mathrm{~mol} / \mathrm{l}$ ) mixed with monomeric and agglomerated metal $(\mathrm{Ag}, \mathrm{Al}, \mathrm{Ni})$ nanoparticles placed in a $140 \mu \mathrm{m}$-thick optical cell and illuminated by 0.532 laser wavelength. The diameters of individual nanoparticles are in the range $10-15 \mathrm{~nm}$ and the agglomerate sizes vary from $100 \mathrm{~nm}$ to about $1000 \mathrm{~nm}$ with the volume fraction of $2 \%$. Our results indicate strong enhancement of lasing intensity from the agglomerated nanoparticle mixtures in comparison with that from single metal nanoparticles and pure R6G solution. The energy threshold for stimulated dye luminescence in solu- tions with agglomerated $\mathrm{Ag}$ and $\mathrm{Al}$ nanoparticles is about $2.4 \mathrm{~mJ}$. This is approximately 30 -times lower than lasing threshold in a pure R6G dye and about four times lower than lasing in mixtures with monomeric $\mathrm{Ag}$ or agglomerated $\mathrm{Ni}$ nanoparticles. We explain our findings by anomalous local optical pump-field enhancement near the complicated surface of nanostructured aggregates.

\section{Acknowledgements}

The authors acknowledge the financial support of Education and Science Ministry of Russian Federation, the register No. 2.4219.2011.

\section{REFERENCES}

[1] D. S. Wiersma, "The Physics and Applications of Random Lasers," Nature Physics, Vol. 4, 2008, pp. 359-367. http://dx.doi.org/10.1038/nphys971

[2] M. A. Noginov, G. Zhu, M. Bahoura, C. E. Small, C. Davison, J. Adegoke, V. P. Drachev, P. Nyga and V. M. Shalaev, "Enhancement of Spontaneous and Stimulated Emission of a Rhodamine 6G Dye by an Ag Aggregate," Physical Review B, Vol. 74, No. 18, 2006, Article ID: 184203. http://dx.doi.org/10.1103/PhysRevB.74.184203

[3] V. M. Markushev, V. F. Zolin and Ch. M. Briskina, "Powder Laser," Zhurnal Prikladnoi Spektroskopii, Vol. 45, 1986, pp. 847-850.

[4] W. L. Sha, C. H. Liu and R. R. Alfano, "Spectral and Temporal Measurements of Laser Action of Rhodamine 640 Dye in Strongly Scattering Media," Optics Letters, Vol. 19, No. 23, 1994, pp. 1922-1924.

http://dx.doi.org/10.1364/OL.19.001922

[5] N. M. Lawandy, R. M. Balachandran, A. S. L. Gomes and E. Sauvain, "Laser Action in Scattering Media," Nature, Vol. 368, 1994, pp. 436-438. http://dx.doi.org/10.1038/368436a0

[6] R. M. Balachandran, D. P. Pacheco and N. M. Lawandy, "Laser Action in Polimeric Gain Media Containing Scattering Particles," Applied Optics, Vol. 35, No. 4, 1996, pp. 640-643. http://dx.doi.org/10.1364/AO.35.000640

[7] W. Kim, V. P. Safonov, V. M. Shalaev and R. L. Armstrong, "Fractals in Microcaviies: Giant Coupled Multiplicative Enhancement of Optical Responses," Physical Review Letters, Vol. 82, No. 24, 1999, pp. 4811-4814. http://dx.doi.org/10.1103/PhysRevLett.82.4811

[8] H. Ramachandran, "Mirrorless Lasers," Pramana, Vol. 58, No. 2, 2002, pp. 313-322.

[9] H. Cao, "Lasing in Random Media," Waves Random Media, Vol. 13, No. 3, 2003, R1-R39. http://dx.doi.org/10.1088/0959-7174/13/3/201

[10] S. V. Karpov, A. L. Bas'ko, A. K. Popov, V. V. Slabko and T. George, "Optics of Nanostructured Fractal Silver Colloids," In: S. G. Pandalai, Ed., Recent Research Developments in Optics, Kerala, 2002, pp. 427-463.

[11] L. A. Sweatlock, S. A. Maier, H. A. Atwater, J. J. Penninkhof and A. Polman, "Highly Confined Electromag- 
netic Fields in Arrays of Strongly Coupled Ag Nanoparticles," Physical Review, Vol. 71, No. 23, 2005, Article ID: 235408.

[12] V. A. Olejnikov, N. V. Pervov and B. V. Mchedlishvili, "Track Membranes in Template Synthesis of SERS-Active Nanostructures," Critical Technologies. Membranes, Vol. 4, 2004, pp. 17-28.

[13] H. C. van de Hulst, "Light Scattering by Small Particles," John Wiley\& Sons Ltd., New York, 1957.

[14] M. Lerner and V. Shamansky, "Synthesis of Nanoparticles by High-Power Laser Pulses," Journal of Structural
Chemistry, Vol. 45, No. 1, 2004, pp. 111-114. http://dx.doi.org/10.1007/s10947-006-0104-3

[15] M. N. Pankratova and V. N. Izmailova, "Structure Formation of Casein Gels," Colloid Journal, Vol. 38, 1976, pp. 490-495.

[16] A. Zhdanov, M. P. Kreuzer, S. Rao, A. Fedyanin, P. Ghenuche, R. Quidant and D. Petrov, "Detection of Plasmon-Enhanced Luminescence Fields from an Optically Manipulated Pair of Partially Metal Covered Dielectric Spheres," Optics Letters, Vol. 33, No. 23, 2008, pp. 2749-2751. http://dx.doi.org/10.1364/OL.33.002749 